municated by the to the Clinical Society in 1879 , where cavernous sounds were audible over a far larger surface than the size of the cavity, owing to its peculiar form. The entire failure of antiseptic inhalations, the knowledge of an adherent pleura, and the certainty of speedy death from septicæmia, induced me to have recourse to the operation, with the immediate results of which I was quite satisfied. I would specially draw attention to the advantage of making an incision, as Mr. Marshall did, over two intercostal spaces in cases where the area of gurgle extends as far; so that, if puncture in the first space fails, we can try the one below or above, as the case may be, without fresh incisions. Another point is using Dr. Hicks' admirable combination of trocar, cannula, and director, ${ }^{1}$ so that the channel into the cavity may not be lost through accident-a by no means infrequent occurrence,-but gradually enlarged with the knife, and a large drainage-tube introduced. The wonderful improvement in the cough, the diminution of the expectoration, and disappearance of fetor, indicated in this case, as in Dr. Douglas Powell's case recorded in the 63rd volume of the Medico-Chirurgical Transactions, that much of the expectoration was due to the irritation set up in the bronchi by the passage of fetid secretion; and the marked amelioration made us hope that we were getting over our difficulties. But the return of fetor, the diminution of the expectoration, and, lastly, the pain in the head, followed by hemiplegia, showed clearly that absorption of matter and pyæmia had set in. The presence of an abscess in the brain and the total absence of any other abscesses in other organs were remarkable, and made one doubt at first if the brain abscess were pyæmic, but the curious relation between the diminution of expectoration and the increase of pain in the head certainly pointed to a pyæmic origin. The expediency of operating at all in these cases must be judged of by considering their probable termination. Some patients appear to gain expectorating power by using antiseptic expectorants in the form of medicines as inhalations, and are enabled to empty their bronchi sufficiently to prevent large and fetid accumulations. In others one of four events happens : (1) Either some of the fetid secretion passes during the movements of respiration into adjoining bronchi, or into the main bronchus of the opposite lung, and gives rise to septic pneumonia and death; (2) or the fetid products are absorbed by the lymphatics and bloodvessels, and give rise to pyæmic abscesses in some part of the body, as in the present case; (3) or, again, the bronchiectasis may burst into the pleura and cause pneumothorax ; (4) or, lastly, the patient may die of suffocative bronchitis from the secretion penetrating into all or the greater part of the alveoli. All these four terminations are fatal in a shorter or longer period, and it is to obviate them that operations are undertaken. Taking the forms of bronchiectasis into account, of the two cylindrical and globular, the globular form of dilatation of the bronchi is the worst to treat, because all tonicity of the walls appears to have been entirely lost, and although we may tap and relieve one of these collections of fetid pus, we never can be quite sure that another one does not exist in another part of the lung, so that not one but many operations are required, and it looks sometimes as if there was more probability of our reaching the end of the patient's life than of the end of the operations. In the three other cases of bronchiectasis on which I have had operations performed, in all more than one operation was performed. In one six openings, in another three, were made, and this last patient stood the operations well, and is still living, a year and a half after. In the present case we were fortunate enough to hit the largest of the bronchiectases, and I hoped, as it was the lowest, that it would carry out sufficient drainage of a large portion of the bronchial tree. The question may be asked why I did not have another operation performed, but we had no indications. The opening was not blocked, but patent, and the septic symptoms came on too rapidly to allow of more apertures being made.

I Lately exhibited at the Medical Society of London.

AT a recent meeting of the guardians of the Wandsworth and Clapham Union, Dr. Longstaff, in moving that the whole question of the accommodation provided in the infirmary and the new workhouse be referred to a committee, is reported to have stated that the infirmary was so overcrowded that some of the inmates had to sleep on the floor and in the kitchen.

\section{ON SPINA BIFIDA.}

\author{
By G. B. BARRON, M.D., M.R.C.S.
}

The subject of spina bifida has been discussed of ilate in the columns of THE LANCET, and an unusual "amount of interest has been evinced in its radical cure by the recognised methods of treatment. How far all cases ray be found to be amenable to any treatment, or how far it is always justifiable to attempt so dangerous an experiment as that of injecting a cyst of this character, is yet beyond the domains of our knowledge. The cases of successful procedure are too limited to warrant any inconsiderate rashness in such a bold and heroic step as rushing in with some badly defined hope of success to stop the gap Nature in her fickle freak has omitted to close. The following case is a fitting one to record as illustrating how tolerant the nerve centres may be of unnatural processes, and how nerve functions may remain unaffected, contrary to our preconceived ideas of their integrity. The literature of hydrorachis is unsatisfactory, as authors, in treating of the subject, pass it over in a brief and rather off-hand manner. The best article on the disease is to be found in Couper's Surgical Dictionary. Cooper relates some interesting cases, but anything like scientific lines of treatment wheren to act with confidence are even here wanting. I apprehend our best surgeons are divided in their opinions as to the most reliable method of dealing with it, and none yet dare issue a dictum of probable successful operation. Cooper relates one case which was cured by repeated tappings and pressure, but states in other cases these measures failed and death ensued. He also says, "We must regard all attempts to cure the disorder by making any kind of opening as exceedingly perilous, and generally fatal." Bryant writes in doubt. He says, "Almost all these cases prove fatal. Many of the subjects are ill-developed, and die within a few days of birth; some die in convulsions, and this mode of death is very usual when the sac bursts and its fluid contents escape." Then, as to treatment, he goes on to say: "Palliative treatment is all that can be followed in the majority of cases, although in exceptional instances operative interference promises to be of use." Holmes somewhat favours injection of the cyst, but this practice is almost only adapted to small pedunculated cases. Erichsen relates a case with an enormously large base, which was successfully treated by tapping. Probably the case he relates has no parallel in surgical literature; but he concludes, "We shall probably best consult the welfare of the child by abstaining from all operative interference." $\mathrm{He}$ does not even allude to injection.

The cases related at the Clinical Society, and the conse. quent discussion, prompt the inference that surgeons are at present most disposed to rely upon injection of the cyst. Still, the meagre success and the paucity of cases so treated scarcely justify reliance upon that method. Here is a void which surgical science requires to deal with without delay.

Three years ago, being on a visit in Worcestershire, I was requested to see the child of a farm labourer living in the adjacent village. The child, well nourished and healthy. looking, was six weeks old, and was the subject of a spina bifida, situated between the fourth and fifth lumbar vertebræ. It was the size of a small hen's egg. Mr. Woodward, of Pershore, had attended the case, and was present at the time I saw it. The skin looked shiny and red, and appeared ready to give way. I advised aspiration or tapping, and the latter procedure was adopted by a large hypodermic syringe needle, which was left in the sac for a few hours to drain. A bout eight drachms of fluid were withdrawn. A compress of cotton-wool was applied to protect the part, and the mother was cautioned not to handle the child roughly, and to avoid undue pressure on the swelling

By the courtesy of Mr. Woodward, in August last I had an opportunity afforded me of seeing the child again. My notes of its condition, taken at the time, run as follows The child is fairly well nourished, and is about the height and weight of a three-year-old child, and has the appearance of full health. It can walk, but its gait is a little hesitating and slightly "straddling," with its feet rather more apart than natural, as though it had a difficulty to secure its equilibrium; the lower extremities are thin, but by no means emaciated, and sensation is perfect, the patellar 
reflex being intact, and plantar titillation immediately evokes the usual muscular jerk. There is perfect control over the sphincters, and the urine is voided in a full stream. There is no lack of intelligence, the eyes are full of expression, the head of normal size and shape, with no sign of hydrocephalus. Pressure on the tumour produces no symptom of distress, nor does it alter materially the size or shape. It has a very broad base, and the edges of the spinal aperture can be felt, the finger-tip detecting a large irregular ovoid opening communicating with the spinal cavity. The size of its base indicates its non-pedunculated character. The measurement in circumference at base is seventeen inches, and across from side to side eleven inches. It is as transparent as a hydrocele. The case, when first seen, did not encourage the idea of attempting a cure by injection, and I may mention that it has been tapped frequently, but has rapidly refilled each time.

The curious and interesting points about this case of hydrorachis seem to me to be these: the size of the sac; the full development of the lower extremities; the power of progression; the absence of pressure on the spinal cord, and thus the sphincter integrity. While the size of the opeving would point to the possible pressure on the cord. If brodd based tumours are more likely to press upon the cord than pedunoulated ones, how is it there is no evidence of pressure here? Suppose the nerve structures are carried furward in front of the flu'd, I think we might reasonably on physiological grounds expect to find evidence of nerve presiure, or malautrition from nerve stretching, or from nerve atrophy. There is no talipes in this case. We are told not to puncture the front part of these tumours so as not to injure the nerves, but is there anv evidence that the puncture of nerve structure in this part of the body by a fine needle has been productive of permanent mischief? This case seems to me, if not unique, at least curious and prompts the following considerations. If the subarachnoid fluid both of the brain and spinal canal normally does not excerd two ounces, how is it that this tumour is so large? We know the fluid is reproduced rapidly when withdrawn, and the withdrawal when comulete produces symptoms analogius to plethora of the b'oodvessels, and when it is in excess it also produces convulsions and other evidences of pressure. There being direct communication between the spinal subarachnoid channel, and the cerebral ventricles, how is it that when the fluid was all drawn away in this case no convulsions ensued? and this procedure has been observed several times without mishap. Does this part of the enveloping sac membrave from its large area alone serete the fluid, or does the whole arachnoidal surface contribute its proportion? and if so why in such excess above normality? The question may also be prompted, Why is there usually talipes in these cases? Is that condition purely accidental or is it due to defective tissue growth dependent upon the spinal abnornality cutting off nerve-supply to a given set of muscles? I confess my inability to suggest any better method of treatiog, radically, hydrorachis; but, except in the matter of injection, we have made no advance in our knowledge of it during the last fifty years. This is a reproach to our modern surgery, which boasts, and with perfect justice, of feats so brilliant that they become almost incredible as compared with the surgery of the past half century. Of this I am quite convinced, that something will dawn upon the intellect, as a more certain and effective means of dealing with this disease. An interest has sprung up which will eventuate in some satisfactory operation. Can we in any way jmitate -loughing which produces spontaneous cure? To say that bydrorachis is an incurable disease would be considered unsurgical, but I am by no means convinced that some better method may not yet be discovered than we at present alopt. The old plan of a seton must be condemned, so I think must tapping and pressure, experience teaching that no reliable good ensues from these measures. It is to be boped every successful case of cure by injection, no matter what the agent used may be, will be published, so that tabulated results may lay the foundation of something like a sound basis whereon to act, and which will inspire confidence in dealing with this bête noire of surgery. Southport.

ON Wednesday morning Dr. Edwardes, of Hounslow, was found dead in his chair. It is stated that there was evidence present of death having been caused by prus:ic acid.

\section{CASE OF FEIGNED SKIN DISEASE.}

\section{By T. COLCOTT FOX, M.B. LoND.}

\section{A. S-, aged nearly sixteen years, a furtive-looking} general servant in London, presented herself at the Skin Department of the Nor:h.West London Hospital on the 28th of November. The catamenia commenced at the age of thirteen, but had since been irregular, and for the past year absent. She was faily well nourished, but pastylooking, and her finger.nails were markedly grooved. She applied on account of three excoriated patches which appedred on Nov. 21st, close togetber on the front of her left leg, and had, she stated, given her gre at pain, and cuused her to lie awake sobbing at night, so that her mistress had no longer the heart to keep her at work, The suggestion had been made that the sores were caused by the dye from her black stockings, and the girl said that she had dressed the places with "Moore's ointment." One sore was perfectly oval, another neally heart-shaped, and the third triangular, with a horg at each angle at the base. The patches measured about $1 \frac{1}{4}$ in. by $1 \frac{1}{2}$ in. to 2 in., and the long axis was in the direction of the limb; they were simple uniform weepi"g excoriations, with hardly any attendant inflammation. Su-pecting the nature of the lesion, I ordered a simple dressing and a saline aperient, and talked of admitting the girl into the hospital if more sores appeared. On Dec. 5th she returned with a transversly oval sore just above each mamma (a very characteristic site in feigned cases, but not quite symmetrically situated. There were two others above the left ankle-joiut, and one on the outside of the right calf, all longitudinally oval simple excoriations, with a welldefined border, except on one sore, where the abrasion was not complete at one end, and only papulation existed, suggesting a cantharides application. There was a dark-brown blood scab on some. She was admitted to the wards. On Dec. 6th a transveistly oval patch appeared just below the left mamma; and another, longitudinally oval, covered with a slight scab, on the right hip. The girl from time to time, for twenty-four hours after admission, had prolonged fits of hysterical sobbing. On the 12th a sore appeared on the right shin near the ankle, and another on the right mamma, between the site of a former excoriation and the areola. There was a remarkable uniformity in the size of the patches throughout. As to the agency at work, I never could detect any evidence of the formation of a bulla, and the patches were too superficial for causation by an acid; moreover, repeated examination of the clothes, bedding, \&c., at convenient tinies and unbeknown to the patient, failed to furnish any clue. On the $16 \mathrm{~h}$, no more excoriations having appeared, I taxed the girl with producing the eruption artificially, and, after prolonged denials, she confessed that she had done so, partly by her nails, but mostly by continued rubbing with the tops of her fingers. Probably the malingering was not al together motiveless, and she desired a rest from her household labours. She proved to be very troublesome and disobedient in the ward, and I learnt from her father that she was an incorrigibly bad girl and a con. stant source of worry. The case is of iuterest as establishing an agency which has hitherto only been suspected as possible. Whether the skin in these cases is peculiarly sensitive to injury is a point for further investigation.

Harley-street.

\section{LARGE ASYLUMS FOR THE INSANE.}

BY H. RAYNER, M.D.,

SUPERINTENDENT OF THE MALE DEPARTMENT OF THE HANWELL COUNTY ASYLUM.

IT appears to me to be desirable to draw attention to a danger which threatens the welfare of the insane poor of England and Wales. This danger arises from the conversion of asylums of a size that is efficient and economic, into larger asylums that are less economic, and I fear less efficient. Moreover, valuable opportunities are being lost by the building of large, cheap asylums for the detention of lunatics, in place of efficient hospitals for their treatment c c 2 